This item was submitted to Loughborough's Research Repository by the author.

Items in Figshare are protected by copyright, with all rights reserved, unless otherwise indicated.

\title{
On the application of laser vibrometry to translational and rotational vibration measurements on rotating shafts
}

PLEASE CITE THE PUBLISHED VERSION

http://dx.doi.org/10.1016/j.measurement.2003.04.001

PUBLISHER

(C) Elsevier Ltd.

VERSION

AM (Accepted Manuscript)

LICENCE

CC BY-NC-ND 4.0

REPOSITORY RECORD

Rothberg, Steve, and John R. Bell. 2019. "On the Application of Laser Vibrometry to Translational and Rotational Vibration Measurements on Rotating Shafts”. figshare. https://hdl.handle.net/2134/8882. 
This item was submitted to Loughborough's Institutional Repository (https://dspace.lboro.ac.uk/) by the author and is made available under the following Creative Commons Licence conditions.

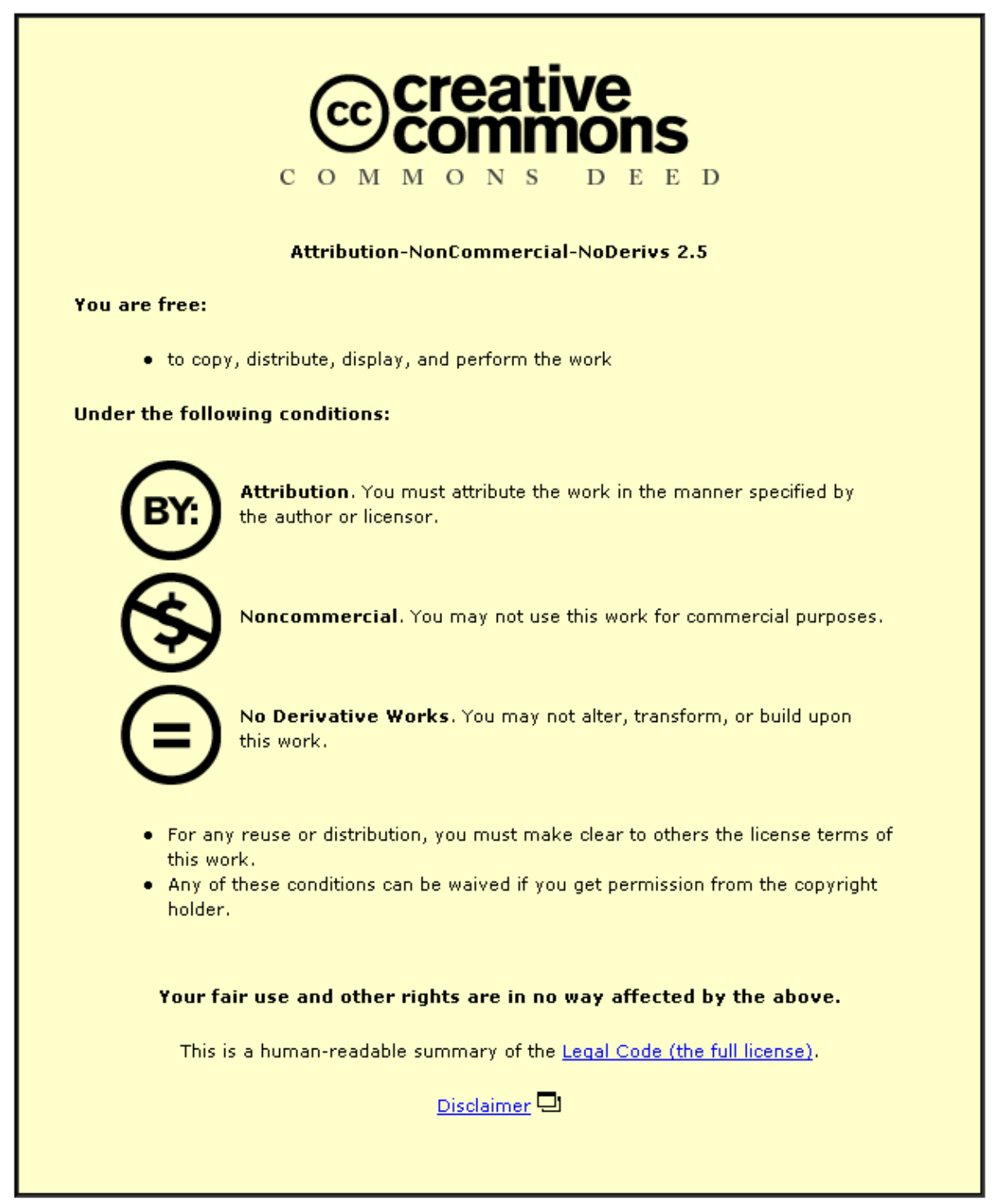

For the full text of this licence, please go to: http://creativecommons.org/licenses/by-nc-nd/2.5/ 


\title{
On the Application of Laser Vibrometry to Translational and Rotational Vibration Measurements on Rotating Shafts
}

\author{
Dr Steve Rothberg, John Bell \\ Wolfson School of Mechanical and Manufacturing Engineering \\ Loughborough University \\ Loughborough \\ Leicestershire LE11 3TU \\ UK
}

\begin{abstract}
Laser Doppler Vibrometry is now a well established experimental technique and commercially available instruments are gaining in popularity. This paper sets out a comprehensive model of the velocity sensed by both a single laser beam and by a pair of parallel beams incident on a rotating shaft requiring three translational and three rotational coordinates to describe its vibratory motion fully. While the intention is to measure the six vibration components, the model reveals how it is instead only possible to measure six vibration "sets", each inseparable combinations of more than one of the vibration components. Arrangements for isolation of each "set" are detailed and an essential postprocessing technique to resolve translational and rotational vibration components is demonstrated in a measurement of crankshaft bending vibration.
\end{abstract}

Keywords: Laser Vibrometry, vibration measurement, rotating machinery

\section{INTRODUCTION}

The non-contact nature of Laser Vibrometers [1] offers significant advantages over traditional contacting vibration transducers and measurements on hot, light or rotating components are often cited as important applications. On rotating machines, it is most common to measure the vibration transmitted into a non-rotating part of the machine using a contacting transducer such as an accelerometer but low vibration transmission can make this unreliable, especially for rotor torsional vibrations. For translational or rotational vibrations, non-contact transducers capable of measuring directly from any location on a structure but especially directly from the rotor itself would be valuable assets and LDV offers this possibility.

Thirty years ago, one of the first reported LDV applications was for axial vibration measurement directly from a rotating turbine blade [2]. Investigations of magnetic discs [3, 4], bladed discs [5, 6] and modal analysis on rotating discs [7] are typical and more recent examples of measurements that can be made using a single probe laser beam. Parallel beam arrangements have also been developed, first for torsional vibration measurement on rotors [8] and more recently for angular vibration measurement on non-rotating structures [9]. Such devices have also been used recently for assessment of crankshaft bending vibration [10].

Previous experience with LDV measurements on rotors has highlighted a cross-sensitivity to vibration components perpendicular to the component it is intended to measure. A feature of much previous work has been prediction of acceptable performance but only in the presence 
of a single vibration component, neglecting the effects of other components present in the more complex motions likely to be encountered in practice. Previous studies have acknowledged cross-sensitivities $(10,11)$ but these have merely been special cases of the totally general case. This paper summarises an analysis of the velocity sensed when laser beams are incident in an arbitrary direction on a target that is of substantial interest in engineering - a rotating shaft requiring three translational and three rotational co-ordinates to describe its vibratory motion fully. To demonstrate the type of cross-sensitivities encountered, measurements are made on the crankshaft of a running diesel engine with the intention of resolving pitch and yaw vibration from simultaneous measurements which individually show cross-sensitivity to pitch in an intended yaw measurement and vice-versa. This resolution uses a post-processing technique, also suitable for radial vibration measurements [12].

\section{VELOCITY MEASURED BY A SINGLE LASER BEAM INCIDENT ON A ROTOR}

The case considered is that of a rotating shaft, which can have arbitrary shape, undergoing an arbitrary vibration requiring three translational and three rotational co-ordinates for description. The resulting theory, however, will be equally applicable to any non-rotating, vibrating structure. As shown in Figure 1, the origin of the translating reference frame $x y z$ is fixed to a point on the centre line of the shaft with the undeflected shaft rotation axis defining the direction and position of the $z$-axis. The time dependent unit vector $\hat{z}_{R}$ defines the changing direction of the shaft rotation axis, which deviates from the $z$-axis as the shaft tilts. $\mathrm{P}$ is the instantaneous point of incidence of the laser beam on the shaft and is identified by the position vector $\vec{r}_{P}$. Provided that the illuminated axial element of the shaft can be assumed to be rigid, the velocity of $\mathrm{P}, \vec{V}_{P}$, is the sum of the translational velocity of origin $\mathrm{O}$ and the velocity of $\mathrm{P}$ relative to $\mathrm{O}$ as a result of rotation about an instantaneous rotation axis passing through O. $\vec{V}_{P}$ can be expanded as:

$$
\vec{V}_{P}=(\dot{x} \hat{x}+\dot{y} \hat{y}+\dot{z} \hat{z})+\dot{\theta}_{x}\left(\hat{x} \times \vec{r}_{p}\right)+\dot{\theta}_{y}\left(\hat{y} \times \vec{r}_{p}\right)+\dot{\theta}_{z}\left(\hat{z} \times \vec{r}_{p}\right)+\Omega\left(\hat{z}_{R} \times \vec{r}_{p}\right)
$$

where $\dot{x}, \dot{y}$ and $\dot{z}$ are the translational vibration velocities of the origin $\mathrm{O}$ in the $x, y$ and $z$ directions (defined by the unit vectors $\hat{x}, \hat{y}$ and $\hat{z}$ ), $\Omega$ is the total rotation speed of the axial shaft element (combining rotation speed with any torsional vibration) and $\dot{\theta}_{x}, \dot{\theta}_{y}$ and $\dot{\theta}_{z}$ are the angular vibration velocities of the shaft around the $x, y$ and $z$ axes (pitch, yaw and roll).

In the usual configuration, a laser vibrometer measures target velocity at the point of incidence in the direction of the probe laser beam given by the unit vector $\hat{b}$. As the shaft vibrates and rotates, however, the position of this point, both on the target and in space, changes continuously, particularly if the shaft is not cylindrical. While the point of incidence does change position in space, it will always lie somewhere along the line of the beam itself and, by this means, an important feature of operation can be demonstrated. The velocity sensed by a laser beam is insensitive to the shape of the target [13], even when the incident beam changes axial and radial position on the target shaft quite dramatically.

The velocity measured by the laser vibrometer, $U_{m}$, is the component of the velocity of the point of incidence in the direction of the incident beam. With the incident beam oriented according to the angles $\alpha$ and $\beta$ (refer to Figure 2), this velocity is given by [13]: 


$$
\begin{aligned}
U_{m} & =\cos \beta \cos \alpha\left[\dot{x}+\left(\dot{\theta}_{z}+\Omega\right) y-\left(\dot{\theta}_{y}-\Omega \theta_{x}\right) z\right] \\
& +\cos \beta \sin \alpha\left[\dot{y}-\left(\dot{\theta}_{z}+\Omega\right) x+\left(\dot{\theta}_{x}+\Omega \theta_{y}\right) z\right] \\
& -\sin \beta\left[\dot{z}-\left(\dot{\theta}_{x}+\Omega \theta_{y}\right) y+\left(\dot{\theta}_{y}-\Omega \theta_{x}\right) x\right] \\
& -\left(y_{0} \sin \beta+z_{0} \cos \beta \sin \alpha\right)\left[\dot{\theta}_{x}+\Omega \theta_{y}\right] \\
& +\left(z_{0} \cos \beta \cos \alpha+x_{0} \sin \beta\right)\left[\dot{\theta}_{y}-\Omega \theta_{x}\right] \\
& +\left(x_{0} \cos \beta \sin \alpha-y_{0} \cos \beta \cos \alpha\right)\left[\dot{\theta}_{z}+\Omega\right]
\end{aligned}
$$

Scanning configurations [7] where the laser beam orientation is a function of time can also be accommodated in this model and this will be a priority for future work.

Equation (2) allows the vibration engineer to be sure of vibration component sensitivity for any laser beam arrangement on any target. It shows that the measured velocity is the sum of six terms, each the product of a combination of geometric parameters and a combination of motion parameters - the "vibration sets". The six vibration sets, in square brackets, are inseparable combinations of different motion parameters and, no matter how a laser beam is aligned, only the sets can be measured directly. The six vibration sets in equation (2) are referred to by the vibration parameter that might be regarded as the intended measurement. These are, in the order presented, the $x$ radial, $y$ radial, axial, pitch and yaw vibration sets and the rotation speed set which includes torsional vibration. Equation (2) can be simplified by setting $z_{0}=0$ so that the plane of the origin of the $x y z$ axes and the "measurement plane" are coincident, since this is just a matter of definition.

A previous description of this cross-sensitivity in radial vibration measurements [11] demonstrated how the "error terms" in the measured velocity, principally $\left(\dot{\theta}_{z}+\Omega\right) x$ and $\left(\dot{\theta}_{z}+\Omega\right) y$ in equation (2), could be of sufficient magnitude to mask the intended radial vibration measurement. A particular problem in the measurement of synchronous radial vibrations was also highlighted. Since the first description of this problem, there has been discussion about whether a particular arrangement of laser beams or a particular variation of the arrangement, for example by scanning the laser beams, might enable resolution of individual motion components. Equation (2) shows that direct measurement of pure radial, axial or bending vibration is not possible because the measurement will always be sensitive to other motion components. It may be possible to assume the effects of additional shaft motions are negligible, enabling direct measurement. For example, if the amplitudes of the vibration components are known to be similar then the intended measurement dominates at frequencies much higher than rotation frequency. In a general case, however, reliable estimation of components requires post-processing as set out in section 4. In contrast, unambiguous measurement of the axial element's time-resolved rotation speed appears possible, accepting that the torsional vibration and roll motion of the shaft are indistinguishable. Isolation of any one of the six sets requires appropriate choice of values for $\alpha, \beta, x_{0}$, and $y_{0}$. A radial and an axial vibration measurement are shown below as examples.

To measure the $x$ radial vibration set requires alignment of the laser beam so that it passes through the centre of the shaft and along the $x$-axis making $\alpha=\beta=0^{\circ}$ and $y_{0}=0$ : 


$$
U_{m}=\left[\dot{x}+\left(\dot{\theta}_{z}+\Omega\right) y-\left(\dot{\theta}_{y}-\Omega \theta_{x}\right) z\right]
$$

Similarly, $\alpha=90^{\circ}, \beta=0^{\circ}$ and $x_{0}=0$ enable the $y$ radial vibration set to be isolated. Equation (3) shows agreement with previous two dimensional theory for radial vibration measurements [11] which was validated experimentally over a range of vibration amplitudes, frequencies and shaft rotation speeds, including speed fluctuations. This equation, however, extends the theory to include motion of the shaft in all six degrees-of-freedom, revealing a third term in the measured velocity, $\left(\dot{\theta}_{y}-\Omega \theta_{x}\right) z$. Attempts to resolve $x$ and $y$ radial motions by post-processing [12] currently rely on the assumption that this third term is an order of magnitude smaller than the first two.

To measure the axial vibration set, aligning the laser beam so that it is parallel to the shaft rotation axis $\left(\beta=270^{\circ}\right)$, makes the measured velocity equal to:

$$
U_{m}=\left[\dot{z}-\left(\dot{\theta}_{x}+\Omega \theta_{y}\right) y+\left(\dot{\theta}_{y}-\Omega \theta_{x}\right) x\right]+y_{0}\left[\dot{\theta}_{x}+\Omega \theta_{y}\right]-x_{0}\left[\dot{\theta}_{y}-\Omega \theta_{x}\right]
$$

Alignment such that $x_{0}$ and $y_{0}$ are zero simplifies the measurement.

\section{MEASURING ROTATIONAL VIBRATION SETS WITH PARALLEL BEAMS}

While values for the geometric coefficients in equation (2) can be found that enable the radial and axial vibration sets to be isolated, isolation of the rotational vibration sets requires the geometric coefficients of the three translational vibration sets to equal zero i.e. $\cos \beta \cos \alpha=\cos \beta \sin \alpha=\sin \beta=0$, to which there is no solution. This means that none of the rotational vibration sets can be isolated using a single laser beam. Repeated application of equation (2) for two beams indicates that isolation of each rotational vibration set requires use of parallel beams. In an interferometer, the difference velocity, $\Delta U_{m}$, is obtained conveniently. For rotation, $\vec{\omega}$, about an instantaneous axis passing through $\mathrm{O}, \Delta U_{m}$ can be written concisely as [14]:

$$
\Delta U_{m}=\vec{\omega} \cdot(\vec{d} \times \hat{b})
$$

where $\vec{d}$ is a vector in the plane of the beams perpendicular to the beam direction $\hat{b}$ and of magnitude equal to the perpendicular separation of the beams, $d$. Figure 2 shows the laser beam orientated according to the angles $\alpha$ and $\beta$ from which $\hat{b}$ can be expanded into Cartesian components. For $\vec{d}$, consider an initial position in the $y z$ plane inclined at an angle, $\gamma$, to $\hat{z}$ as shown in Figure 3. After finite rotations, first by $\beta$ and then by $\alpha$, the projection of $\vec{d}$ into each direction can be found. Finally, expanding $\vec{\omega}$ in the manner of equation (1), equation (5) can be written as:

$$
\begin{aligned}
\Delta U_{m}=d\left[(\sin \gamma \sin \beta \cos \alpha-\cos \gamma \sin \alpha)\left[\dot{\theta}_{x}+\Omega \theta_{y}\right]\right. & \\
& \left.+(\sin \gamma \sin \beta \sin \alpha+\cos \gamma \cos \alpha)\left[\dot{\theta}_{y}-\Omega \theta_{x}\right]+\sin \gamma \cos \beta\left[\dot{\theta}_{z}+\Omega\right]\right]
\end{aligned}
$$

Table 1 summarises the configurations enabling isolation of each of the rotational vibration sets. Where considerations such as limited access do not allow the necessary alignment, 
consideration must be given to the influence of other motions on the intended measurement. A previous study [15] highlighted the sensitivity to angular vibrations of rotation speed set measurements made with the Laser Torsional Vibrometer (LTV) when $\beta \neq 0$. A maximum angle of $20^{\circ}$ was recommended.

Note the sensitivity to both pitch and yaw in the pitch and yaw sets. This is an important problem that can only be addressed by post-processing as described in section 4 .

\subsection{Measurement using twin pairs of parallel laser beams}

In many practical situations access to the shaft is restricted and it is not physically possible to align the beams at the angles set out in the previous section to isolate the required vibration set. The requirement for just one or two distinct values of $\alpha$ or $\beta$, rather than the possibility to measure at any orientation is very restrictive. For example, with this restriction, measurement of unambiguous rotation speed cannot be achieved from the end face of a shaft which is often the only area of access. An alternative optical arrangement capable of measuring the shaft rotation speed, pitch vibration and yaw vibration sets from a variety of angles is desirable. The geometric values required to measure the vibration sets using two pairs of parallel beams are summarised in Table 2 and depicted in Figures 4a-c.

Three basic beam configurations exist; in the first, the beams are incident on the side of the shaft while, in the second, the beams are incident on the end face of the shaft. As shown in Figures $4 \mathrm{a} \& \mathrm{~b}$, the desired angular vibration set is selected by choosing the appropriate value for $\alpha$, while measurement of the rotation speed set can be achieved with any value of $\alpha_{1}=\alpha_{2}=\alpha$. This means that, for the same physical arrangement, measurements of the rotation speed set and one of the angular vibration sets can be obtained simultaneously simply by choosing to take both the sum, $\Sigma(\Delta U)$, and the difference, $\Delta(\Delta U)$, in the velocities measured by each individual pair of beams. In the third configuration shown in figure $4 \mathrm{c}$, the beams are incident on the side of the shaft at different axial locations. This arrangement enables simultaneous measurement of the pitch and yaw vibration sets by taking both the sum and the difference in the velocities measured by each individual pair of beams.

\section{POST-PROCESSING FOR RESOLUTION OF VIBRATION COMPONENTS}

This post-processing technique is equally applicable to radial and angular vibration measurements. It is written in angular terms to support the crankshaft pitch and yaw measurements that follow. The pitch, $\dot{\Theta}_{x}(t)$, and yaw, $\dot{\Theta}_{y}(t)$, vibration sets can be written:

$\dot{\Theta}_{x}(t)=\dot{\theta}_{x}+\Omega \theta_{y}$

$\dot{\Theta}_{y}(t)=\dot{\theta}_{y}-\Omega \theta_{x}$

It is possible to manipulate equations $(7 \mathrm{a} \& \mathrm{~b})$ to give the genuine angular vibration velocities,

$\dot{\theta}_{x}$ and $\dot{\theta}_{y}$, on a frequency-by-frequency basis in terms of the measurable quantities $\dot{\Theta}_{x}(t)$, $\dot{\Theta}_{y}(t)$ and $\Omega$. Evaluating for the $m^{\text {th }}$ component i.e. at $\omega=\omega_{m}$ gives:

$\dot{\theta}_{y_{m}}=\left.W\left(\omega_{m}\right)\left(\dot{\Theta}_{y}(t)+\Omega \int \dot{\Theta}_{x}(t) d t\right)\right|_{\omega=\omega_{m}}$ 
where $W\left(\omega_{m}\right)=\left(\omega_{m}^{2} / \omega_{m}^{2}-\Omega^{2}\right)$. Similarly, considering the pitch vibration:

$$
\dot{\theta}_{x m}=\left.W\left(\omega_{m}\right)\left(\dot{\Theta}_{x}(t)-\Omega \int \dot{\Theta}_{y}(t) d t\right)\right|_{\omega=\omega_{m}}
$$

Therefore, the genuine vibration velocity components can be resolved by evaluating the bracketed terms of equations $(8 \mathrm{a} \& \mathrm{~b})$ and then weighting by $W\left(\omega_{m}\right)$. Inspection of equations $(8 \mathrm{a} \& b)$ shows that the weighting term is infinite and the bracketed term is zero for synchronous vibrations, i.e. $\omega_{m}=\Omega$, which makes this technique unsuitable for synchronous vibration measurement. This leads to a gap in the resolved data at the synchronous frequency and a velocity-time trace cannot be reconstructed. This is not a limitation of the postprocessing technique but a limitation on the use of LDV for both translational and rotational vibration measurements on rotors. At synchronous frequency, equations ( $7 \mathrm{a} \& \mathrm{~b})$ are no longer independent and cannot be solved as simultaneous equations. A measured synchronous component can only be considered as a conservative estimate of the sum of the two (either angular or radial) velocities.

\section{PITCH AND YAW METER}

In order to make simultaneous measurements of pitch and yaw vibration, eliminating the rotation speed set, a novel optical configuration was devised. The configuration shares basic features with the laser tiltmeter [9] and is shown in Figure 5a. A rotating diffraction grating, used to provide the frequency shifts needed for directional discrimination, provides a convenient means of deriving three beams. The $+1,0$ and -1 diffracted orders are used giving three beams shifted by $+0.8 \mathrm{MHz}, 0$ and $-0.8 \mathrm{MHz}$ from the HeNe laser source. The lens L1 focuses the beam onto the diffraction grating and the diffracted beams are made parallel by lens L2. Two small mirrors, M1 and M2, are then used to steer the -1 order into the beam arrangement shown in Figure 5b. A polarising beamsplitter and quarter wave plate are used to increase the light efficiency by maximising both incident light on the target and scattered light routed to the photodetectors. Non-polarising beamsplitters NPBS1, NPBS2 and NPBS3 and mirrors M3, M4 and M5 are then used to combine the 0 and -1 orders onto detector 1 and the 0 and +1 orders onto detector 2 where they beat. Mirrors M3 and M4 are also used to compensate path length imbalances introduced in creating the 3-beam arrangement. The pitch and yaw meter is designed for use on the end face of a shaft and the three parallel laser beams are aligned with the shaft spin axis. Combination of beams 1 and 2 gives the pitch vibration set and combination of beams 1 and 3 gives the yaw vibration set after demodulation of the detector outputs.

The pitch and yaw halves of the laser instrument were calibrated, one at a time, by making the beams incidents on a small test rotor, rotating at (nominally) constant speed, with the plane of the beams perpendicular to the rotor's rotation axis. A very convenient independent measurement of angular velocity is provided by the pseudo-random noise in the instrument output caused by the laser speckle phenomenon [16]. This noise introduces peaks in the demodulated output spectrum at the fundamental rotation frequency and integer multiples from which rotation speed can be easily obtained. Voltage output per unit angular velocity was thus provided. 
These peaks remain present during any measurement directly from a rotating target and this can be of some significance when attempting to measure at low vibration levels at integer multiples of shaft rotation frequency. The effect has been given the name pseudo-vibration.

\subsection{Diesel Engine Crankshaft Vibration Measurement}

The pitch and yaw meter was aligned parallel to the crankshaft rotation axis of a four cylinder two litre diesel engine and incident on the end face of the crankshaft pulley to provide simultaneous measurements of the pitch and yaw vibration sets. In addition, a Laser Torsional Vibrometer (LTV) was used to measure the crankshaft rotation speed. The beams of the LTV were aligned as close to perpendicular to the rotation axis as access would allow to eliminate the sensitivity of that measurement to bending vibration.

The measurement could also be performed by an engineer using two of the Laser Rotational Vibrometers that are commercially available. As viewed from the front of the engine, one of the beam pairs would appear horizontal and the other beam pair would appear vertical. Whether a custom optical configuration is used or whether commercial instruments are used, it is still necessary to resolve the pitch and yaw vibrations from the raw measurements taken using the method of section 4 .

Both pitch and yaw vibrations were resolved and Figures 6a\&b show, as examples, waterfall plots of the resolved crankshaft pitch and yaw vibrations, respectively, across a range of engine speeds, obtained by varying engine load (the engine was driving a water brake) while maintaining wide open throttle. The individual resolved spectra show the pattern of peaks at integer multiples of half engine orders, as expected for a four-stroke engine. The vibration levels encountered in the pitch measurement exceed those in the yaw measurement which results from the manner in which the combustion load is applied to the crankshaft. The plots show lines of RPM vs. frequency for second engine order and its integer multiples up to tenth engine order, as these are the excitation orders at which greatest response is expected in a four-stroke, four-cylinder engine. At higher speeds and higher engine orders, the peaks broaden indicating that the engine speed was varying during the course of the spectrum averaging undertaken. In the future, this could be improved upon by use of an order analysis. Note how there is a gap in the resolved data at synchronous frequency, as discussed in section 4 , which means that it is not possible to reconstruct pitch and yaw time signals. Other regions of the spectra can, however, be reconstructed and, in any case, first engine order is not a particularly important frequency for a four-stroke, four-cylinder engine. The broad peak around $450 \mathrm{~Hz}$ is a particularly significant feature in the data. Earlier modal tests on the stationary crankshaft [10] had indicated this to be the frequency of the first bending mode of the crankshaft which occurs in the range $456-475 \mathrm{~Hz}$ depending on the angular position of the stationary crankshaft. Response around this frequency range can be seen in the pitch data even at the lowest RPM but the response grows considerably as the eighth and tenth engine orders close in on this frequency range at higher engine speeds. The ability to make a quantitative investigation of these kinds of features on a running and un-modified engine underlines the value of LDV as a means to provide vibration data in the most challenging of circumstances.

\section{CONCLUSIONS}

This paper has summarised theory describing the velocity sensed by laser beams incident on a rotating structure requiring three translational and three rotational co-ordinates to describe its vibratory motion fully. The theory is equally applicable to measurements on targets with 
simpler motions, such as non-rotating targets, allowing the vibration engineer to determine the vibration component sensitivity of any measurement with any laser beam orientation. Timedependent laser bean orientation can also be included in the model, extending its application to scanning Laser Vibrometry.

Six separate vibration sets, each a combination of motion parameters, appear in the full expression for the vibration velocity sensitivity using a single laser beam. The $x$ radial, $y$ radial, and axial sets can be isolated by appropriate geometric set-up of the laser beam while the pitch and yaw vibration sets and the rotation speed set can be eliminated but not isolated with a single laser beam. Parallel beam arrangements can isolate the rotational vibration sets, offering insensitivity to the translational vibration sets. A full range of versatile arrangements using two pairs of parallel beams was also presented as an alternative means to measure the pitch, yaw and rotation speed sets. All measurements demonstrate insensitivity to target shape.

Previous analyses have predicted satisfactory application when only single vibration components have been considered. For a rotating shaft undergoing arbitrary motion, direct measurement of radial or axial vibration is not possible because the measurement will always be sensitive to other motion components. Resolution of individual motion components within each set has been shown not to be possible by any geometric arrangement of the laser beam or by introduction of additional laser beams. An approximate solution to resolve steady-state, non-synchronous, vibrations was applied to the assessment of pitch and yaw vibration of the crankshaft of a running diesel engine enabling identification of the first natural frequency in bending. As the first such non-contact measurements, the potential of laser technology for machinery diagnostics under challenging conditions is, once again, emphasised.

\section{ACKNOWLEDGEMENTS}

The authors acknowledge the support of the Engineering and Physical Sciences Research Council.

\section{REFERENCES}

1. D.C. WILLIAMS (ed.) 1993 Optical methods in engineering metrology Chapman \& Hall, London, Chapter 6.

2. Q.V. DAVIS and W.K. KULCZYK 1969 Nature 222, 475-476. Vibrations of turbine blades measured by means of a laser.

3. T.A. RIENER, A.C. GODING and F.E. TALKE 1988 IEEE Transactions on Magnetics 24(6), 2745-2747. Measurement of head/disc spacing modulation using a two channel fiber optic laser Doppler vibrometer.

4. R.W. WLEZEIN, D.K. MIU and V. KIBENS 1984 Optical Engineering 24(4), 436442. Characterization of rotating flexible disks using a laser Doppler vibrometer.

5. R.A. COOKSON and P. BANDYOPADHYAY 1980 Transactions of the ASME Journal of Engineering for Power 102, 607-612. A fiber-optics laser Doppler probe for vibration analysis of rotating machines.

6. A.K. REINHARDT, J.R. KADAMBI and R.D. QUINN 1995 Transactions of the ASME Journal of Engineering for Gas Turbines and Power 117, 484-488. Laser vibrometry measurements on rotating blade vibrations. 
7. A.B. STANBRIDGE and D.J. EWINS 1995 Proceedings of ASME Design Engineering Technical Conference, Boston, USA 3(B), 1207-1213. Modal testing of rotating discs using a scanning LDV.

8. N.A. HALLIWELL, C.J.D. PICKERING and P.G. EASTWOOD 1984 J. Sound and Vibration 93(4), 588-592. The laser torsional vibrometer: a new instrument.

9. N.A. HALliWELL, A HOCKNELL and S.J. ROTHBERG 1997, J. Sound and Vibration 208(3) 497-500. On the measurement of angular vibration displacements: a laser tiltmeter.

10. T.J. MILES, M. LUCAS, N.A. HALLIWELL and S.J. ROTHBERG 1999 Journal of Sound and Vibration 226(3), 441-467. Torsional and bending vibration measurement on rotors using laser technology.

11. S.J. ROTHBERG and N.A. HALLIWELL 1994 Transactions of the ASME Journal of Vibration and Acoustics 116(3), 326-331. Vibration measurements on rotating machinery using laser Doppler velocimetry.

12. J.R. BELL and S.J. ROTHBERG 1998 Proceedings of SPIE - 3rd International Conference on Vibration Measurements by Laser Techniques: Advances and Applications, Ancona, Italy 3411, 14-22. Radial vibration measurements on rotors using laser vibrometry: a first practical solution to the cross-sensitivity problem.

13. J.R. BELL and S.J. ROTHBERG 2000 Journal of Sound and Vibration 237(2) 245261. Laser vibrometers and contacting transducers, target rotation and 6 degree-offreedom vibration: what do we really measure?

14. J.R. BELL and S.J. ROTHBERG 2000 Journal of Sound and Vibration 238(4) 673690. Rotational vibration measurements using laser vibrometry: comprehensive theory and practical application.

15. T.J. MILES, M. LUCAS and S.J. ROTHBERG 1995 Proceedings of the 15th ASME Biennial Conference on Vibration and Noise, Boston, USA, 84-3(C) 1451-1460. The laser torsional vibrometer: successful operation during lateral vibrations.

16. S.J. ROTHBERG, J.R. BAKER and N.A. HALLIWELL 1989 Journal of Sound and Vibration 135(3) 516-522. Laser Vibrometry: Pseudo-Vibrations 
$\underline{\text { Vitae }}$

Dr Steve Rothberg is Head of the Wolfson School of Mechanical and Manufacturing Engineering at Loughborough University. He has research interests in noise and vibration measurement and analysis, especially the development of laser transducers for vibration measurement.

John Bell was a research student at Loughborough University between 1996 and 1999, gaining a $\mathrm{PhD}$ degree for his work on applications of laser Vibrometry to rotor vibration measurements. He now works for Ilmor Engineering Ltd. 
Figure legends

Figure 1: Definition of a point $\mathrm{P}$ on a vibrating and rotating component undergoing arbitrary vibration.

Figure 2: Orientation of laser beam, defining incident angles $\alpha$ and $\beta$.

Figure 3: Orientation of parallel beam separation defining angle $\gamma$.

Figure 4a: Configurations of pairs of parallel laser beams for measurement of pitch and rotation speed sets.

Figure 4b: Configurations of pairs of parallel laser beams for measurement of yaw and rotation speed sets.

Figure 4c: Configurations of pairs of parallel laser beams for measurement of pitch and yaw sets.

Figure 5a: The pitch and yaw meter optical configuration.

Figure 5b: The pitch and yaw meter beam pattern on the target.

Figure 6a: Resolved pitch vibration.

Figure 6b: Resolved yaw vibration. 
$\underline{\text { Tables }}$

\begin{tabular}{|l|c|c|c|l|}
\hline Desired measurement & $\alpha$ & $\beta$ & $\gamma$ & $\begin{array}{l}\text { Positioning of beams } \\
\text { on shaft (side or end) }\end{array}$ \\
\hline Pitch set, $d\left(\dot{\theta}_{x}+\Omega \theta_{y}\right)$ & 0 & $\pm \pi / 2$ & $\pi / 2$ & End \\
\cline { 2 - 5 } & $\pi / 2$ & $\beta$ & 0 & Side \\
\hline Yaw set, $d\left(\dot{\theta}_{y}-\Omega \theta_{x}\right)$ & $\pi / 2$ & $\pm \pi / 2$ & $\pi / 2$ & End \\
\cline { 2 - 5 } & 0 & $\beta$ & 0 & Side \\
\hline Rotation speed set, $d\left(\dot{\theta}_{z}+\Omega\right)$ & $\alpha$ & 0 & $\pi / 2$ & Side \\
\hline
\end{tabular}

Table 1: Optical configurations enabling isolation of each of the rotational vibration sets.

\begin{tabular}{|l|l|l|l|l|l|l|l|l|}
\hline $\begin{array}{l}\text { Desired } \\
\text { measurement }\end{array}$ & Signal & $\alpha_{1}$ & $\alpha_{2}$ & $\beta_{1}$ & $\beta_{2}$ & $\gamma_{1}=\gamma_{2}$ & $\begin{array}{l}\text { Positioning of } \\
\text { beams on shaft } \\
\text { (side or end })\end{array}$ & Figure \\
\hline$\dot{\theta}_{x}+\Omega \theta_{y}$ & $\Delta(\Delta U)$ & 0 & 0 & $\beta$ & $-\beta$ & $\pi / 2$ & Side & $4 \mathrm{a}$ \\
\cline { 2 - 9 } & $\Sigma(\Delta U)$ & 0 & 0 & $\beta$ & $\pi-\beta$ & $\pi / 2$ & End & $4 \mathrm{a}$ \\
\cline { 2 - 9 } & $\Delta(\Delta U)$ & $\alpha$ & $-\alpha$ & 0 & 0 & 0 & Side & $4 \mathrm{c}$ \\
\cline { 2 - 9 } & $\Sigma(\Delta U)$ & $\alpha$ & $\pi-\alpha$ & 0 & 0 & 0 & Side & $4 \mathrm{c}$ \\
\hline \multirow{3}{*}{$\dot{\theta}_{y}-\Omega \theta_{x}$} & $\Delta(\Delta U)$ & $\pi / 2$ & $\pi / 2$ & $\beta$ & $-\beta$ & $\pi / 2$ & Side & $4 \mathrm{~b}$ \\
\cline { 2 - 9 } & $\Sigma(\Delta U)$ & $\pi / 2$ & $\pi / 2$ & $\beta$ & $\pi-\beta$ & $\pi / 2$ & End & $4 \mathrm{~b}$ \\
\cline { 2 - 8 } & $\Sigma(\Delta U)$ & $\alpha$ & $-\alpha$ & 0 & 0 & 0 & Side & $4 \mathrm{c}$ \\
\cline { 2 - 8 } & $\Delta(\Delta U)$ & $\alpha$ & $\pi-\alpha$ & 0 & 0 & 0 & Side & $4 \mathrm{a} \& \mathrm{~b}$ \\
\hline$\dot{\theta}_{z}+\Omega$ & $\Sigma(\Delta U)$ & $\alpha$ & $\alpha$ & $\beta$ & $-\beta$ & $\pi / 2$ & Side & $4 \mathrm{a} \& \mathrm{~b}$ \\
\cline { 2 - 8 } & $\Delta(\Delta U)$ & $\alpha$ & $\alpha$ & $\beta$ & $\pi-\beta$ & $\pi / 2$ & End & \\
\hline
\end{tabular}

Table 2: The geometric values required to measure the vibration sets using two pairs of parallel beams as depicted in Figures $4 a-c$. 